\title{
PERSONALENTWICKLUNG
}

\section{Verantwortung für Berufsbiografien}

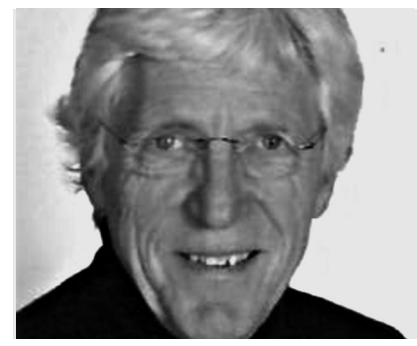

VON WOLFGANG HOFFMANN

Hoffmann (58) ist Dozent an der Bundesakademie für Kirche und Diakonie gGmbH (BAKD) und an der Führungsakademie für Kirche und Diakonie gAG (FAKD). Er ist dort u. a. für die Themen Management in sozialen Organisationen und Personalentwicklung verantwortlich. hoffmann@bundesakademiekd.de
Die demografische Entwicklung wird die Personalsituation auf dem deutschen Arbeitsmarkt grundlegend ändern. Für manchen sozialen Dienstleister wird sich die Frage stellen, ob er noch die Mitarbeitenden bekommen wird, um seinen Auftrag zuverlässig und kreativ zu erfüllen. Deshalb werden in Zukunft die Unternehmen stärker als bisher Verantwortung für die Berufsbiografien ihrer Mitarbeitenden übernehmen müssen.

Die Bevölkerung in Deutschland wird zunehmend älter. Es werden deutlich weniger junge Menschen dem Arbeitsmarkt zur Verfügung stehen. Bis zum Jahr 2020 wird es mehr über 50-jährige als unter 30-jährige Erwerbstätige geben. Die Sozialwirtschaft muss, wenn sie weiterhin ihren Dienstleistungsauftrag erfüllen will, entsprechende Personalentwicklungs- und Bildungsmaßnahmen einleiten.

Beim genauen Hinschauen wird man jedoch feststellen, dass die zu erwartende Entwicklung ohnehin notwendige und sinnvolle Personal- und Organisationsentwicklungsmaßnahmen nur dringlicher machen und die damit ohnehin notwendigen Prozesse beschleunigen. Die Instrumente dafür liegen meist vor - was oft fehlt, ist das Bewusstsein für die Notwendigkeit von Veränderungen und ein auf die veränderten Rahmenbedingungen bei der Belegschaft abgestimmtes Konzept für folgende Situation:

- Es wird immer weniger gut ausgebildetes Personal für die Leistungserbringung in der Sozialwirtschaft zur Verfügung stehen.

- Das vorhandene Personal wird in $\mathrm{Zu}$ kunft deutlich länger arbeiten müssen. Die heute fast durchgängig genutzte Altersteilzeit wird auslaufen und die Regelarbeitszeit ist bereits auf 67 Jahre heraufgesetzt. Die Unternehmen sind gezwungen, den »Marktwert « ihres Personals über einen längeren Zeitraum zu erhalten. Das zielt nicht nur die körperli- che Leistungsfähigkeit; viel wichtiger wird es sein, die Motivation für die Arbeit für einen längeren Zeitraum zu erhalten und die Fachlichkeit auf Dauer den Erfordernissen des Aufgabenbereichs anzupassen.

Schon ab dem Jahr 2010 haben 50-jährige Mitarbeitende noch bis zu 17 Jahre zu arbeiten! Während sich in den letzten Jahren viele Mitarbeitende ab dem 50. Lebensjahr auf den vorzeitigen Ruhestand ab etwa dem 58. Lebensjahr als Perspektive eingerichtet haben, wird es in Zukunft notwendig sein, 50-jährige Mitarbeitende im Rahmen der Personalentwicklung darauf anzusprechen, welche Perspektiven sie sich für die kommenden 17 Berufsjahre vorstellen. Mit 50 Jahren muss das Potenzial in den Menschen nochmals neu entdeckt und gefördert werden. Neue Bildungsangebote und Personalund Organisationsentwicklungskonzepte müssen sich an dieser Herausforderung orientieren.

Es sind im wesentlichen die Mitarbeitenden, die über den Erfolg oder Misserfolg eines Unternehmens entscheiden. Das Personal ist das entscheidende Leistungspotenzial in der dienstleistungsorientierten Sozialwirtschaft. Das folgende Potenzial muss als Ressource in Zukunft rund 45 Jahre erhalten und gefördert werden: Talent und Begabung im Umgang mit Menschen, Freude und Motivation an der Ausübung der Tätigkeit, Fachlichkeit, Engagement und Innovation, Erfahrung, Gesundheit.

Die Ausprägung dieses Potenzials bestimmt den »Marktwert « der Mitarbeitenden. Es ist also naheliegend, dass sowohl die Mitarbeitenden als auch das Unternehmen ein großes Interesse daran haben, diesen »Marktwert Personal « über einen Zeitraum von 45 Jahren zu erhalten. Für Führungskräfte wird dies die entscheidende Herausforderung der kommenden Jahre sein.

Den Mitarbeitenden muss klar sein, dass es keine Nischen mehr geben wird, in denen man sich ab dem 50. Lebensjahres bis zum Erreichen des (Vor-) Ruhestandes einrich- 
ten kann. Nur ein hoher, für den Arbeitgeber attraktiver »Marktwert « der Arbeitsleistung ist Voraussetzung für ein ausreichend hohes Leistungspotential und damit für eine Sicherung des Einkommens.

Das Unternehmen braucht leistungsstarke und motivierte Mitarbeitende um erfolgreich seinen Dienstleistungsauftrag erfüllen zu können. Immer weniger junge Menschen werden dem Arbeitsmarkt zur Verfügung stehen. Die Mitarbeitenden über 50 Jahre bleiben als Leistungsträger unverzichtbar. Die Investition in dieses Potenzial ist für die Unternehmen keine Kann-Leistung, sondern ein Pflichtaufgabe!

Der Erhalt und die Erweiterung der Potenziale der vorhanden Mitarbeitenden kommt auf strategischer Ebene somit eine grundlegende Bedeutung zu. Sowohl Mitarbeitende als auch die Träger müssen fortlaufend darauf achten, schlummerndes Potenzial zu wecken und es für das Unternehmen nutzbar zu machen. Kontinuierliche Bildungsangebote erhalten den Marktwert der Mitarbeitenden als »Kapital « des Unternehmens.

Schauen wir uns beispielhaft die Phasen eines zukünftigen Berufslebens an. Dafür nehme ich eine Person, die mit 25 Jahren die pädagogische Ausbildung beendet hat und direkt nach der Ausbildung eine Stelle in der stationären Jugendhilfe antritt.

\section{Phase: 1. bis 5. Berufsjahr}

Mit hohem Engagement und ausrechender fachlicher Grundqualifikation werden die Grundanforderungen erfüllt. Die Person ist leistungsbereit, hoch motiviert und engagiert. Nach zwei Jahren wird die Befristung des Arbeitsverhältnisses aufgehoben. Gleichzeitig wird verabredet, dass ergänzend zur Grundqualifikation zusätzliche Fachkompetenz wünschenswert ist. Um den »Marktwert « der Person zu erhöhen, bieten sich folgende Fortbildungen an (noch keine Zusatzqualifikation als Spezialisierung): klientenzentrierte Gesprächsführung und Deeskalationstraining. Diese Investition wird sich lohnen. Der »Markwert « der Person ist hoch, sie ist auch noch nach fünf Berufsjahren für jeden Arbeitgeber attraktiv.

\section{Phase: 6 . bis 15 . Berufsjahr}

Nach fünf Berufsjahren in der stationären Jugendhilfe muss eine Bilanz gezogen werden:

- Bist du in diesem Bereich der Jugendhilfe an der richtigen Stelle?

- Entsprechen die Anforderungen deiner Fachlichkeit?

- Willst du auch die nächsten zehn Jahre mit diesem Personenkreis arbeiten?
- Wie stellst du dir die nächsten zehn Jahre Deines Berufslebens vor?

Es stehen entscheidende Weichenstellungen an. Um das »Kapital Arbeitskraft « der Beispielperson für das Unternehmen weiter effektiv nutzen zu können, muss in die Bildung investiert werden. Diese Investition erhöht gleichzeitig den "Marktwert " des gesamten Personalbestandes. Stellen beide Partner jetzt fest, dass der Bereich der Jugendhilfe auch für die nächsten zehn Jahre die richtige Entscheidung ist, muss eine Erweiterung der fachlichen Kompetenzen erfolgen. Eine Spezialisierung als Zusatzausbildung zur Fachkraft für »Traumaerkennung und Traumabehandlung " kann an dieser Stelle die richtige Entscheidung sein. Mit der Erweiterung der Fachlichkeit wird gleichzeitig im laufenden Personalentwicklungsprozess die Phase der Karriereplanung (andere Funktion, einbinden in Projekte, erweitere Zuständigkeitsbereiche o. Ä.) beginnen. Mögliche alternative Entscheidungen können zu diesem Zeitpunkt aber auch sein:

- Die Person entscheidet sich für ein anderes Arbeitsfeld. Ein Wechsel steht an. Fluktuation als Chance für beide Seiten! - Die Partner sind unsicher, ob eine Perspektive von zehn Jahren angebracht ist. Alternativen werden erwogen. Die Entscheidung für eine Spezialisierung und einer Karriereplanung entfällt. Stattdessen wird der "Markwert " der Person durch laufende, nicht ausschließlich zielgruppenspezifische Fortbildungen erhalten. Nur gut ausgebildete Mitarbeitende sind attraktiv für andere Arbeitgeber!

Egal wie die Berufsbiografie im Einzelfall weiter verläuft; in jedem Fall muss weiter in Erhalt und Verbesserung der Fachkompetenz investiert werden. Nach 15 Berufsjahren ohne laufende Ergänzung der Grundqualifikation und ohne Veränderung der beruflichen Perspektiven sinkt der » Marktwert « einer Fachkraft erheblich. Die Motivation und damit die Leistungsbereitschaft lässt nach. Die Person wird auch für andere Arbeitgeber zunehmend unattraktiv. Wo das Personal das entscheidende Leistungspotenzial ist, muss sich der Träger dann den Vorwurf gefallen lassen, den Wert genau dieses Potenzials Mitarbeitende nicht ausreichend bewahrt und gefördert zu haben.

\section{Phase: 16 . bis 25. Berufsjahr}

Unsere Beispielperson ist jetzt 40 Jahre alt. Wenn die Personalentwicklung gut gelungen ist, haben wir es mit einer Person zu tun, die über eine Zusatzausbildung verfügt, aktiv in Veränderungsprozesse einbezogen wurde und Erfahrungen in mindestens einem weite- ren Arbeitsfeld der Jugendhilfe hat. Diese Person ist jetzt auf dem Gipfel ihres »Markwertes «. Folgende Fragen geben jetzt Aufschluss über die weiteren Perspektiven:

- Entspricht die derzeitige Aufgabe und Funktion den Erwartungen?

- Sind berufliche Perspektiven bei diesem Träger realistisch erkennbar?

- Ist das Arbeitsfeld der Jugendhilfe auch für die nächsten zehn Jahre attraktiv?

- Sind berufliche Perspektiven in anderen Arbeitsfeldern (bei einem anderen Träger) denkbar und wünschenswert?

- Entspricht die Qualifikation und Fachlichkeit den anzustrebenden Berufsperspektiven?

Grundsätzliche berufliche Veränderungen und Entwicklungen müssen jetzt eingeleitet werden. Dies ist der richtige Zeitpunkt für mögliche Neuorientierung beruflicher Perspektiven. Schlummernde Potenziale können gefördert und für das Unternehmen nutzbar gemacht werden. Werden weitergehende Führungstätigkeiten angestrebt, muss eine Managementausbildung geplant werden. Bei einer Veränderung des Tätigkeitsfeldes im Umfeld der Jugendhilfe (bei dem gleichen Träger) sind zusätzliche Kompetenzen notwendig. Ist ein Wechsel in ein Arbeitsfeld außerhalb der Jugendhilfe wünschenswert, sind die entsprechend notwendigen Qualifikationen zu organisieren.

Für ein Unternehmen kann es langfristige sehr attraktiv sein, zum richtigen Zeitpunkt auch in Bildungsangebote zu investieren, die ein Wechsel einzelner Mitarbeitender zu einem anderen Träger ermöglichen. Investition in Fluktuation als Konzept!

\section{Phase. 26. bis 42. Berufsjahr}

Wir sind bei dem genannten Beispiel angekommen. Unsere Person ist jetzt 50 Jahre alt und hat 25 Jahre Berufserfahrung in der Jugendhilfe hinter sich. Bestenfalls hat sie sich laufend qualifiziert, hat sich durch Zusatzausbildungen spezialisiert, ist nicht mehr im stressigen Gruppendienst eingebunden und war im bisherigen Berufsleben aktiv an Veränderungsprozessen beteiligt. Bisher haben Unternehmen in dieses »Humankapital « der über 50-Jährigen kaum noch investiert. Auch das Interesse der Mitarbeitenden an Bildungsangeboten lässt in dieser Phase deutlich nach. Berufliche Perspektiven rücken deutlich in den Hintergrund. Der Erhalt des Geleisteten hat Priorität. Da ein Ausscheiden aus dem Berufsleben vor dem 67. Lebensjahr in Zukunft aber ausgeschlossen ist, stellen sich folgende Fragen:

- Wie willst du die nächsten 17 Berufsjahre verbringen? 
- In welchem Bereich willst du nochmals "durchstarten "

- Welche deiner Potentiale wurden bisher zu wenig oder gar nicht für das Unternehmen genutzt?

- Was kannst du heute besser als vor 15 Jahren?

- Welche Strukturen im Unternehmen verhindern oder behindern dein Engagement?

- Was möchtest du in Zukunft nicht mehr machen?

Da zu diesem Zeitpunkt ein Wechsel zu einem anderen Träger - eventuell in ein anderes Arbeitsfeld - eher unwahrscheinlich ist, bleibt unsere Beispielperson die nächsten 17 Jahre auf der Lohnliste des Jugendhilfeträgers. Es muss also in seinem Interesse sein, diese Ressource für sein Unternehmen wei- ter zu nutzen. Jetzt profitieren Arbeitgeber und Arbeitnehmer von einer bisher gelungenen Personal- und Bildungsentwicklung. Die Person ist gut ausgebildet, hat eine gute Beziehung zum Unternehmen, ist motiviert und an neuen Aufgaben interessiert. $17 \mathrm{Be}$ rufsjahre sind allemal genug Grund, neue Perspektiven zu entwickeln und weiter in die Bildung für diese Person zu investieren.

\section{Perspektiven und Anregungen}

Nein, es gibt keinen » Königsweg «. Es gibt keine Patentrezepte und keine Checklisten für die anspruchsvolle Gestaltung von zukünftigen Berufsbiografien. Es gibt aber eine Wahrheit: Wer sich nicht ab sofort mit diesem Thema beschäftigt und die passenden Stellschrauben für einen wertschätzen- den Umgang mit den Mitarbeitenden im Unternehmen sucht, wird es in spätestens zehn Jahren auf dem konkurrierenden Sozialmarkt sehr schwer haben, seinen Dienstleistungsauftrag erfüllen zu können. In Zukunft werden die Unternehmen viel stärker Verantwortung und Engagement für die Berufsbiografien ihrer Mitarbeitenden übernehmen müssen.

Dabei wird es notwendig sein, mit 50jährigen Mitarbeitenden im Rahmen der Personalentwicklung nochmals ganz neue Perspektiven zu erarbeiten. Mit 50 Jahren muss das Potenzial dieser Menschen neu entdeckt und gefördert werden. Bildungsangebote, Konzepte der Personalentwicklung und des betrieblichen Gesundheitsmanagement und die Arbeitsbedingungen müssen sich an dieser Herausforderung orientieren.

\section{Was Sozialunternehmen jetzt tun müssen}

Die demografische Entwicklung und die Verlängerung der Lebensarbeitszeit erfordern von sozialen Organisationen und von Unternehmen der Sozialwirtschaft einen neuen Umgang mit Mitarbeiterinnen und Mitarbeitern. Vier Aspekte sind dabei von herausragender Bedeutung:

1. Individuelle Lösungen als Konzept. Das Prinzip der »individuellen Lösungen« sollte zum Konzept erklärt werden. Jede Berufsbiografie orientiert sich neben den Anforderungen der Unternehmen primär an den Bedürfnissen, Fähigkeiten, Macken und individuellen Eigenschaften der Mitarbeitenden. So individuell, wie die einzelnen Menschen sind, so individuell werden in Zukunft die Berufsbiografien sein, insbesondere $a b$ dem 50. Lebensjahr. Gleichmacherei als Maßstab für einen "gerechten « Umgang mit allen Mitarbeitenden wird den zukünftigen Erfordernissen nicht mehr gerecht. Mit jeder Einzelperson muss eine individuelle Startbahn geplant werden, die ein nochmaliges berufliches Durchstarten mit 50 ermöglicht. Lassen Sie niemanden in ihrer oder seiner Nische zurück!

2 Personalentwicklungskonzepte neu gestalten. In Zukunft wird eine erfolgreiche Personalentwicklung daran zu messen sein, ob es gelingt, Berufsbiografien gezielt zu steuern und dabei Potenziale laufend zu entdecken und zu fördern. Die Arbeitskraft und Motivation des Personalbestandes über einen Zeitraum von 45 Berufsjahren als das entscheidende (Human-) Kapital zu entwickeln und zu erhalten gilt als die große Herausforderung für Führungskräfte. Bildung und Gesundheit müssen in moderne Konzepte der Personalentwicklung integriert werden. Strategisches Management kommt an der Personalentwicklung nicht mehr vorbei. Personalentwicklung ist der Schlüssel für eine erfolgreiche Unternehmenspolitik.

3. Mitarbeiterpools ermöglichen Gestaltungsspielraum Größere Personaleinheiten ermöglichen einen wünschenswerten Gestaltungsspielraum für Mitarbeitende und Unternehmen. Wechsel von Arbeitsbereichen und Arbeitsfeldern sind nur in größeren Einheiten denkbar. Die langfristige
Qualifizierung von Mitarbeitenden für zukünftige Anforderungen kann in der strategischen Personalentwicklung nur in größeren Einheiten sinnvoll und effektiv geleistet werden. Größere Komplexeinrichtungen haben an dieser Stelle einen Vorteil, müssen diesen aber konsequenter für die Personalentwicklung nutzen. Kleine, oft spezialisierte Einrichtungen oder Träger, die ausschließlich Leistungen in einzelnen Arbeitsfeldern erbringen, kommen schnell an ihre Grenzen. Sie sind wenig flexibel und können keine alternative Arbeitsfelder und damit wenig Gestaltungsspielraum bieten. Die Perspektive, "einmal Jugendhilfe, immer Jugendhilfe«, kann sich als großes Problem bei der Gestaltung von Berufsbiografien erweisen. Größe Einheiten durch Kooperationsverträge mit anderen Trägern in der Region können für Mitarbeitende und Träger attraktive Perspektiven schaffen. Mitarbeitende werden strategisch auf zukünftige Bedarfe geschult, Karriereplanungen sind nicht nur auf kleine Einheiten beschränkt, personale Potenziale können besser genutzt werden. In größeren Einheiten können die Träger auf Befristungen bei den Arbeitsverträgen weitgehend verzichten. Die Mitarbeitenden werden ihnen dies durch Engagement und Verbundenheit danken.

4 Fluktuation als Konzept. Nur gut ausgebildete Mitarbeitende sind auch für andere Arbeitgeber attraktiv. Mitarbeitende, die nur deshalb im Unternehmen bleiben, weil sie an andere Stelle keine Chance haben, sind meist nicht das gewollte Personal. Wenn Sie Ihre Mitarbeitenden attraktiv für andere Träger machen, sind sie auch für Ihr Unternehmen attraktiv. Fluktuation ermöglicht für Ihr Unternehmen eine Neuorientierung. Qualifizieren Sie also auch, um Fluktuation zu ermöglichen. Das ist gut angelegtes Geld. Der Wert Ihres Unternehmens hängt entscheidend von dem "Gesamtmarktwert" Ihrer Mitarbeitenden ab. Mitarbeitende, die nach 15 Berufsjahren in der Jugendhilfe nur deshalb im Unternehmen bleiben, weil sie für andere Bereiche nicht über die notwendige Kompetenzen verfügen, werden sich auf Dauer nicht als die Leistungsträger erweisen. Was spricht also dagegen, in Fluktuation zu investieren?

Wolfgang Hoffmann 